- Distance learning is playing an increasingly important role in continuing professional development.

- A wide range of paper and electronically-based distance learning material is now available for dentists and the dental team.

- These guidelines have been designed to help users to choose appropriate and effective distance learning material.

\title{
Distance learning materials for dentists - a users guide to quality
}

\author{
K. A. Eaton ${ }^{1}$ and M. Hammick ${ }^{2}$
}

\begin{abstract}
In May 2001, an open meeting was held at the BDA headquarters to discuss how the quality of distance learning material for dentists and the dental team could be assured in the future. As a result of the meeting an ad hoc group of users, producers, teachers and researchers was formed. The group have produced this paper which seeks to help dentists and all members of the dental team select quality material relevant to their needs. The President of the General Dental Council has added a foreword to this guide.
\end{abstract}

\section{FOREWORD}

The General Dental Council supports innovation in the provision of continuing professional development (CPD) in terms of delivery, media and access, and welcomes the growth of new methods of dental education.

The Council's Recertification Scheme will form a foundation for the future revalidation of the dental team. The scheme is designed to allow dentists to exercise their own professional judgement about the nature of CPD activity they undertake, encouraging participation in activities beyond traditional methods of educational delivery.

\section{The legal requirement to undertake CPD}

CPD is defined as 'study, training and other activities undertaken by a dentist which he or she reasonably intends should

\footnotetext{
${ }^{1 *}$ Teledentistry Leader and Senior Honorary Research Fellow, Eastman Dental Institute, University College London ${ }^{2}$ Senior Lecturer, Centre for Research in Medical and Dental Education, University of Birmingham.

${ }^{*}$ Correspondence to: Dr Kenneth Eaton, Teledentistry Leader and Senior Honorary Research Fellow, Eastman Dental Institute, University College London, 123 Gray's Inn Road, London WC1X 8WD

Email:K.Eaton@eastman.ucl.ac.uk
}

Received 6.12.02; Accepted 16.12.02

๑ British Dental Journal 2003; 194: 253-256 advance his or her professional development.'

The statutory scheme requires all dentists to undertake 250 hours of CPD over each five year cycle; 75 of these hours must be verifiable CPD. Verifiable CPD does not simply mean that participation can be verified. To be verifiable CPD, an activity must meet the Council's three educational criteria:

1. The activity must have concise educational aims and objectives - the activity should have a clear purpose or goal.

2. The activity must have clear anticipated outcomes - participants should know what they can expect to gain as a result of taking part in the activity.

3. The activity must be subject to quality controls - participants should have an opportunity to give feedback, with a view to improving quality.

In addition to the three educational criteria, dentists will have to be able to prove their participation in the activity by obtaining from the activity provider documentary evidence. This evidence must show the number of CPD hours involved.

The requirements are the same for all forms of learning, including distance learning activities. When deciding whether or not a particular activity meets the statutory requirement for verifiable
CPD, the activity should always be measured against the Council's criteria, and availability of documentary proof of participation, showing the number of CPD hours involved confirmed. It will still be open for the activity to be include in CPD records as general CPD if the requirements for verifiable CPD are not met.

This guide will hopefully assist in exploring opportunities for distance learning and in deciding whether or not a particular distance learning activity will advance professional development. Once an activity has been completed, participants are encouraged to make the most of the opportunity to give feedback on the activity to the provider and to reflect on its value to professional development. Dentists create the market for postgraduate CPD and, as consumers of continuing education, are in a position to influence course provision by making experiences and views known.

The desired outcomes of good CPD choices are: benefits to patients, the advancement of dental teams, and a rewarding experience, professionally.

\section{INTRODUCTION}

This guide aims to help in the assessing of quality of distance (also known as open) learning materials. The guide has been reviewed and endorsed by the organisa- 
tions listed at the end of the document and it is hoped will appear on their web sites. It also appears as an annex to the British Dental Association advice sheet E10, CPD Clinical governance audit and peer review. There is currently considerable expansion in the use of distance learning, in general, and by UK dentists and other members of the dental team, in particular. Distance learning materials have a great advantage, in that they may be used to provide higher education or CPD at anytime and at any place, in contrast to more traditional methods that involve attendance at a specific location, often at a specific time. The recent amendments to the Dentists Act 1984 make CPD throughout a practising lifetime mandatory for all dentists included in the Dentists Register and have provided dentists with a stimulus to use all forms of CPD including distance learning. However, in spite of the interest in, and expansion of, distance learning, little guidance is available to help its users to select quality material relevant to their needs.

\section{AIMS AND OBJECTIVES}

The guide aims to help users, to select the optimum material for their learning needs. It defines distance learning and identifies the three key aspects of distance learning materials and criteria against which quality can be judged. A number of check lists are provided to help to achieve these objectives.

\section{WHAT IS DISTANCE LEARNING?}

Distance learning is any form of learning that takes place without the learner and teacher present in person in the same room. It is particularly useful for anyone who experiences the barriers of time and place which can be encountered with more traditional courses. It can use various forms of old and new technology, including:

- paper-based,

- paper-based plus practical or clinical assignments carried out at home or in the workplace,

- audio-based material (such as tapes, records, radio),

- video-based material,

- computer-based material,

- television.

E-Learning is a rapidly growing aspect of distance learning and uses a range of ICT-based materials such as CD-ROMs, DVDs, video-conferencing, video-streaming (also known as web-casting), internet forums and chat rooms, and dental television channels to deliver learning material. The term distance learning is often interchanged with the term e-learning to reflect the use of the internet and multimedia in course content. E-learning appears to mean different things to different people. To some it is all embracing and represents the future of all learning opportunities. To others it is one more approach in a comprehensive tool kit of learning methodologies that together create new and exciting ways to learn. One thing is certain; in the learning world of today, it cannot be ignored.

Distance learning is often best achieved with a combination of formats: the more traditional print-based materials and the newer e-learning. It is also apparent that many learners find it helpful to have an opportunity to meet their tutors face to face, especially before embarking on a course or other prolonged learning experience.

\section{HOW TO EVALUATE QUALITY}

With the range and technological scope of material now available, many of the more traditional quality standards and criteria are outdated or inappropriate. These guidelines aim to help dentists make an informed choice and to seed their expectations.

Three key aspects of distance learning materials that can be assessed to help find quality course are outlined below. They are educational, scientific (content) and technological aspects. To assess these aspects it is necessary to ask three broad questions, which are set out below.

- Does the material encourage and enable effective learning?

- Are its contents scientifically correct?

- Is the technology user-friendly, reliable and durable?

These questions will now be considered in some detail. But first it is useful to consider two points. One is to check that the distance learning material has been independently assessed (pre-piloted) before distribution to intended users and their comments fed back to the producers. The other is to continually ask the question 'is this material (programme) enjoyable to use?'

\section{DOES THE MATERIAL ENCOURAGE AND ENABLE EFFECTIVE LEARNING?}

Effective learning is dependent on the content, style and activities of the materials provided. Distance learning material should provide ways to assess whether learning outcomes are being achieved. This is done through the assignments and assessments that are set during and at the end of the course. Most learners use distance learning materials on their own. Good tutor and peer support, explicitly stated before the course starts can make a large contribution to its enjoyment and success. It is also vital that participants know from the beginning what resources are needed to complete the programme.
In general, the content should:

- Be coherent, clear and consistent, ie easy to follow.

- Fit in with any wider programme it may be a part of.

- Develop thinking and learning skills, taking into account prior skills, and knowledge.

- Enable participants to reflect on new knowledge and skills in terms of their own professional experience.

- Encourage participants to implement what they have learnt, ie improve practice.

\section{Specifically, good learning materials will} have:

- A statement about their target audience, eg post graduate general dentists.

- Explicit learning outcomes or aims and objectives.

- A short introduction with an overview of the content and an estimated overall time that should be spent on the learning.

- Well-structured content matched to the learning outcomes (or aims and objectives), including activities and reference to further readings and other sources.

- Plans for updating the learning materials to ensure they remain relevant.

\section{High quality material will:}

- Be written simply and clearly, with definitions of any new terminology.

- Be presented in short, manageable chunks.

- Have content (text) interspersed with activities.

- Include suggestions for further reading and opportunities for reflection.

- Avoid sexist or other discriminatory language, and unnecessary jargon.

\section{Activities, assignments and assessment}

should:

- Be achievable and realistic, without using too many resources.

- Generate interest and enthusiasm.

- Relate to the learning outcomes (or aims and objectives).

- Support reflection on new knowledge and skills in terms of professional experience.

- Provide an indication of how long they will take.

- Be followed by some commentary.

- Present different types of problems or questions and require various modes of response.

- Be clearly linked to any award given on completion of the programme.

They should encourage reflection, especially on the links between theory and prac- 
tice and through this, enhance the development of workplace knowledge and skills.

\section{Demonstration courses}

It is recommended that demonstration courses are attended before signing up for a series of programmes or a long course and before committing time (and possibly money).

\section{ARE THE CONTENTS SCIENTIFICALLY CORRECT?}

The material used may be from written or electronic sources and may appear in various formats. One way of assessing their quality is to use skills of critical analysis alongside an accepted hierarchy of strength of evidence, as, for example, reported by Harrison. ${ }^{2}$ In general, expect quality standards to match those of traditional (attendance) courses.

Table 1 shows the diversity of material that delegates may come across and comments on the value and rigour of the different types of information found within the material.

\section{IS THE TECHNIOLOGY USER-FRIENDLY, RELIABLE AND DURABLE?}

This section mainly applies to e-learning materials but some of it will be useful for the more traditional forms of paper-based open learning material. Criteria such as ease of use and technical proficiency are useful overall guides to assessing whether this type of material is as good - or better - than 'being there'. In this way participants can be more confident that it will be a useful and enjoyable learning experience. Table 2 highlights some questions to ask about this type of material.

\section{Finally}

The key features to look for when choosing open learning materials are in the checklist below.

\section{CHECKLIST}

What format does the open learning take?

How will it be updated?

How will feedback be provided?

Can questions be fed back to the providers/technical experts?

Is there a 'live' customer help facility?

Is this verifiable CPD?

How is it verified?

Is it interactive?

What is it that makes it interactive?

What proof of educational standards is provided?

If Internet based, is the video element full screen, full motion video?

Does it display sound learning objectives?

Does it test for retained knowledge?

Does it have high production values?
}

Table 1 diversity of learning materials

\begin{tabular}{|c|c|c|}
\hline Format & Type & Guide to scientific quality \\
\hline \multirow[t]{4}{*}{ Written } & Papers in a refereed journal & $\begin{array}{l}\text { These undergo rigorous peer assessment procedures that } \\
\text { should, in the majority of cases, ensure scientific quality. }\end{array}$ \\
\hline & Books or chapters in books & $\begin{array}{l}\text { This material is peer assessed but in a less rigorous way than } \\
\text { papers in refereed journals. }\end{array}$ \\
\hline & Un-refereed journal articles & These do not generally have any checks for scientific probity. \\
\hline & Handouts or course manuals & $\begin{array}{l}\text { Course material aims for a balance between the scientific rigour } \\
\text { of a published paper and user friendliness. }\end{array}$ \\
\hline Electronic & $\begin{array}{l}\text { Web sites, CD ROMs, Video, } \\
\text { Video conferencing }\end{array}$ & $\begin{array}{l}\text { Good quality material will be: } \\
\text { - Correctly referenced } \\
\text { - Have an indication of the strength of evidence to support a } \\
\text { particular view or procedure } \\
\text { - Wherever possible present the contrary view together with its } \\
\text { - Mupporting evidence }\end{array}$ \\
\hline
\end{tabular}

Table 2 Questions to ask about e-learning materials

\begin{tabular}{l|l}
\hline Questions & Comments \\
\hline
\end{tabular}

Is it possible to provide proof of completion of the learning and to evaluate the material?

This must be more than the start and finish times of your viewing. If it is possible to make a cup of tea, ring a mate, and come back just in time for the end of the programme, then that is, at best, general not verifiable CPD.

Verifiable CPD programmes will have built-in tracking of the activities engaged in. This way confirmation can be obtained of contribution to bulletin boards, chat rooms, and/or completed assignment tasks. Check that the material explains how this will be done and how privacy will be preserved within the tracking system.

Is it possible to comment on whether or not the course was found worthwhile and how it can be improved on?

Tracking could also enable feedback from a tutor, as progress is made through the materials, and can provide on-going evaluation of the material.

It is vital that there is end point evaluation to enable participants to feedback how well the constituent parts of the whole course fitted together.

Does the e-learning use the full strengths of the online medium?

The essence of the online medium is lost if users can sit back and let the information flow over them. Good engagement is more than just turning the pages of the programme. The programme should enable users to become part of a community of learners, around 20 is an ideal size for such a group. In this way the material enables the dialogue capability of the medium to be fully used.

Are delegates encouraged to be an active participant and to interact with the subject matter?

Activities and quizzes at strategic points enable users to discover how their learning is progressing. Immediate feedback will enable them to apply the knowledge immediately.

Regular interactions track progress and can tell users how they have interacted with the material.

Material on CD-ROM should be designed with hot links to the source to

allow transmission of answers and comments back to the source.

Is a genuine multimedia experience offered?

Multimedia e-learning should be visually driven, rather than totally text driven. Full-screen, full motion video with animation, text and music in combination will give a rich learning experience.

Is the learning based on sound principles that will ensure maximum knowledge retention?

Is it possible to tell how much knowledge and understanding has been retained?

The pace of delivery should be appropriate for the material and match users' learning style and previous knowledge.

An immediate test indicates that the information has been retained fo between one minute and the duration of the programme. Retention can only be tested after a suitable time (two to three weeks).

Is there compatibility between web based material and the IT being used.

Is the speed of data transfer between you and the web server satisfactory?

Has the provider used time saving techniques to enable quicker access to large data objects?
Designs that are simple, whilst still being effective, are more likely to suit most IT systems.

Picture files are often better displayed as a small 'thumbnail' of around 5-10kb linked to the larger picture. Users can then click the thumbnail only if they want to see (and wait for) the larger picture.

Centrally maintained, frequently changing, linked text and other relatively small objects that require little bandwidth can live on a web server and be accessed via the world wide web. Full screen, high quality video in real time via the web is currently unavailable for most users because of bandwidth restrictions. However, high speed internet access known as broadband or ADSL is now much more readily available. Techniques such as Web on CD enable large data objects such as printable-quality pictures and full-screen video clips to be put on a distributed CD ROM, so the user can access them quickly. 
A list of web sites on the assessment of web-based material can be found at http://www.vuw.ac.nz/ agsmith/evaln/e valn.htm. The Quality Assurance Agency for England's Guidelines can be found at http://www.qaa.ac.uk/public/dlg/contents.htm. Additional sources are given at the end of this guide and many libraries and information services provide tutorials for this purpose.

\section{REFERENCES}

1 General Dental Council Continuing Professional Development Rules. 2001.

2 Harrison J E. Evidence-based orthodontics - how do I assess the evidence? J Ortho 2000 27: 189 -196.

\section{BIBLIOGRAPHY}

1 ANSI Standards Committee on Dental Informatics, Working Group Educational Software Systems, ANSI/ADA 1001-2002 Guidelines for the Design of Educational Software. 2001.

2 Forsyth I. Teaching and learning materials and the Internet. 3rd Edition. London: Kogan Page, 2002.

3 Johnson L, Schleyer T. Developing standards for the design of educational software. Quintessence Int; 1999 30: 763 - 768.

4 Maier P, Warren A. Integrating technology in learning and teaching - a practical guide for educators. London: Kogan Page, 2001.

5 Oliver R. Paganelli C. Towards a global superstore of quality-assured modularised learning programmes. Report from Section 4.3, DentEd Evolves - Global Congress in Dental Education. Eds. D. Shanley, A Nattestad, R. Valachovic. Eur J Dent Educat2002: 6 (Suppl 3): 5-6.

Thank you to the following people for their contribution to this guide: -

Dr Alison Bullock, Senior Research Fellow, Centre for Research in Medical and Dental Education,

University of Birmingham.

Mr David Bythell, Education Programme Manager, Smile-on.com.

Dr Lester Ellman, General Dental Practitioner and Chairman of the General Dental Practice Committee of the British Dental Association.

Professor John Frame, Regional Director of Postgraduate Dental Education for the West Midlands, Director of the Centre for Research in Medical and Dental Education, The University of Birmingham, Professor of Oral and Maxillo-Facial Surgery, The University of Birmingham.

Dr Richard Oliver, Senior Lecturer and Honorary Consultant in Orthodontics, University of Wales College of Medicine and Leader of the Quality Guidelines for Electronically-based Dental Education Group of the DentEd Evolves Project.

Mr Malcolm Pendlebury -Dental Tutor, Faculty of General Dental Practitioners (UK), Adviser In
Postgraduate Dental Practice and Director of the Clinical Education Masters Programme the University of Nottingham.

Ms Tracey Posner, Marketing Director, Redbus CPD.

Mr Ray Reed, Dental Postgraduate Dean for Wessex, Past Dean of the Faculty of Dental Surgery, Royal College of Physicians and Surgeons of Glasgow and Vice-President of the Senate of Dental Specialties.

Mr Marc Trup, General Dental Practitioner and Managing Director, Redbus, CPD.

Professor Damien Walmsley, Professor of Restorative Dentistry, University of Birmingham.

List of organisation that have approved these guidelines

- British Dental Association

- Council of Postgraduate Dental Deans and Directors

- Faculty of General Dental Practitioners (UK)

- Joint Committee for Continuing Professional Development

- Redbus CPD

- Senate of Dental Specialties

- Smile-on.com

- University of Birmingham

- University of Wales College of Medicine 